Ann. Biol. anim. Bioch. Biophys., I976, 16 (5), 635-643.

\title{
INDUCTION ARTIFICIELLE DE L'OVULATION ET FERTILITÉ CHEZ LA LAPINE (ORYCTOLAGUS CUNICULUS) ALLAITANTE OU NON
}

\author{
Françoise HULOT et B. POUJARDIEL \\ Station d'Amélioration génétique des Animaux, \\ Centre de Recherches de Toulouse, I. N.R. A., \\ 31320 Castanet-Tolosan
}

\section{RÉSUMÉ}

Une étude de l'induction artificielle de l'ovulation par des procédés mécaniques et hormonaux a été réalisée chez des lapines Californiennes nullipares ( 72 femelles) et allaitantes (53 femelles) ; l'examen des ovaires est effectué 7 jours après le traitement. L'excitation mécanique du col utérin conduit parfois à l'ovulation ( 5 femelles sur 33 ). L'injection de HCG (25 et 50 UI) induit la ponte ovulaire avec une fréquence élevée : $97 \mathrm{p}$. 100 chez des femelles nullipares, 82 p. Ioo chez des femelles à I I jours de lactation. Le nombre moyen d'ovules ainsi pondus est comparable à celui obtenu après accouplement chez les femelles nullipares, mais plus faible chez les femelles allaitantes.

Une deuxième expérience portant sur 54 femelles nullipares métisses de races diverses, est consacrée à l'étude de la viabilité in vivo des ovocytes dont la ponte est induite artificiellement par HCG ou LH ovine. Les femelles sont inséminées et abattues 12 jours plus tard pour dénombrer corps jaunes et embryons. Quatre-vingt-dix-huit p. Ioo des femelles ovulent et les valeurs moyennes du nombre d'ovulations et d'embryons sont statistiquement égales dans les deux modes d'induction. Le pourcentage des femelles gestantes est inférieur de I3 $\mathrm{p}$. Ioo au pourcentage de femelles ovulant. La moitié des portées subit des pertes partielles qui représentent I 3,5 p. Ioo des ovules pondus. L'importance de cette mortalité se situe dans les limites de celle obtenue en saillie naturelle.

\section{INTRODUCTION}

I'ovulation chez la Lapine est, dans les conditions naturelles, provoquée par l'accouplement. De nombreuses recherches ont déjà porté sur l'ovulation et sur les procédés capables de l'induire artificiellement. Devant conduire un élevage en insé- 
mination artificielle, nous avons cherché à connaître l'efficacité de certains types d'induction artificielle de l'ovulation sur le nombre d'ovules pondus et sur leur qualité. Nous avons utilisé deux types de traitement : la stimulation mécanique des cols utérins et l'injection hormonale, sur des lapines nullipares et allaitantes.

\section{MATÉRIEL ET MÉTHODES}

Les animaux étudiés étaient élevés sur grillage, en cage individuelle, dans un bâtiment conventionnel clos, avec fenêtres, ventilé et, en période hivernale, chauffé de façon à maintenir une température de $18^{\circ} \mathrm{C}$. La durée du jour, à l'intérieur des cellules est, par éclairage artificiel, maintenue à $\mathrm{I}_{4}$ heures par 24 heures. Les lapines étaient nourries ad libitum avec un aliment granulé complet du commerce dosant $\mathrm{I} 7 \mathrm{p}$. Ioo de matières azotées totales et $12-\mathrm{I}_{4} \mathrm{p}$. I00 de cellulose.

Une première expérience a permis de comparer les résultats d'ovulation de quatre lots de lapines : un lot de lapines accouplées à des mâles vasectomisés, servant de témoin (lot I); des lots avec induction artificielle réalisée soit par excitation des cols utérins au moyen d'une baguette (lot 2), soit par injection intraveineuse de HCG aux doses de 25 UI (lot 3) et de 50 UI (lot 4). Soixante-douze femelles nullipares de souche Californienne élevées au laboratoire, âgées de 4,5 à 5 mois ont été réparties en 18 groupes de 4 femelles d'âge et d'ascendance semblables. Les femelles d'un groupe sont soumises en simultanéité aux 4 traitements. Les traitements ont eu lieu entre le I er février et le I 5 mars I97I. Une laparotomie est effectuée, 7 jours plus tard, pour observer la présence éventuelle de corps jaunes de pseudogestation permettant l'estimation du nombre d'ovulations. Trois à quatre semaines plus tard, toutes les femelles saines sont mises au mâle normal. Onze jours après la parturition, en période d'allaitement, chaque lapine subit le même traitement que précédemment. Les corps jaunes sont dénombrés sept jours plus tard, après abattage.

Une deuxième expérience, réalisée sur des femelles nullipares âgées de 4 à 5 mois, métisses de races diverses, est consacrée à l'étude de la qualité des ovocytes pondus. L'ovulation est induite par une injection intraveineuse de 25 UI d'HCG chez 25 femelles, ou de 10 , 15 ou $20 \mu g$ de LH ovine chez 29 femelles. Chaque animal est inséminé avec $0,5 \mathrm{ml}$ de mélange de sperme frais de deux mâles. Onze jours plus tard les femelles sont abattues, le nombre de corps jaunes et d'embryons est enregistré. Chez les femelles vides il est encore possible de dénombrer les corps jaunes de pseudo-gestation.

Dans les deux expériences, les femelles ont été traitées sans tenir compte du cycle cestrien.

\section{RÉSULTATS}

\section{I. - Induction artificielle de l'ovulation sans insémination}

Deux caractères sont analysés : présence ou non d'ovulation, nombre d'ovulations.

\section{Fréquence de l'ovulation.}

Peu de femelles, tant nullipares (tabl. I) qu'allaitantes (tabl. 2) ovulent après stimulation mécanique du col.

Le nombre de femelles qui ovulent après traitement par HCG (lots 3 et 4) est supérieur à celui observé dans le lot témoin (lot $\mathrm{I}$ ) aussi bien chez les femelles nullipares $\left(\chi^{2}=4,72\right.$; 0,0I0 $\left.<p<0,025\right)$ que chez les femelles allaitantes $\left(\chi^{2}=2,52\right.$; $0,100<p<0,250)$. 
La fréquence de femelles ovulant est plus faible chez les femelles allaitantes ( $0,82 \pm 0,16$ avec HCG et $0,55 \pm 0,32$ dans le lot témoin) que chez les femelles nullipares $(0,97 \pm 0,06$ avec $\mathrm{HCG}$ et $0,78 \pm 0,16$ dans le lot témoin). Ces différences ne sont significatives ni dans le lot témoin $\left(\chi^{2}=1,42\right.$; 0 , I0o $\left.<p<0,250\right)$ ni dans les lots traités avec HCG $\left(\chi^{2}=3,43 ; 0,050<p<0\right.$, IOO) .

\section{TABI,EAU I}

Induction artificielle de l'ovulation femelles californiennes nullipares

(Février-mars I97I)

\begin{tabular}{|c|c|c|c|c|}
\hline \multirow{2}{*}{ Traitements } & \multirow{2}{*}{$\begin{array}{c}\text { Mâle } \\
\text { vasectomisé } \\
\text { Lot } 1\end{array}$} & \multirow{2}{*}{$\begin{array}{c}\text { Excitation } \\
\text { mécanique } \\
\text { Lot } 2\end{array}$} & \multicolumn{2}{|c|}{ Traitements hormonaux } \\
\hline & & & $\begin{array}{c}\text { HCG } 25 \mathrm{UI} \\
\text { Lot } 3\end{array}$ & $\begin{array}{c}\text { HCG } 50 \text { UI } \\
\text { Lot } 4\end{array}$ \\
\hline Nombre de femelles traitées $\ldots$. & 18 & 18 & 18 & 18 \\
\hline Nombre de femelles ayant ovulé ......... & $1 ' t$ & 4 & 17 & 18 \\
\hline Nombre de femelles ayant ovulé $(\%) \ldots \ldots$ & 78 & 29 & 94 & 100 \\
\hline Nombre de corps jaunes..$\ldots \ldots \ldots \ldots \ldots$ & 157 & 39 & 213 & 237 \\
\hline Moyenne corps jaunes $(m) \ldots \ldots \ldots \ldots$ & 11,21 & 9,75 & 12,52 & 13,16 \\
\hline Variance $\left(\sigma^{2}\right)$ & 3,56 & 16,25 & 5,01 & $\begin{array}{l}37,79 \\
\because, 56\left({ }^{1}\right)\end{array}$ \\
\hline
\end{tabular}

(1) Valeurs obtenues en éliminant un cas de super-ovulation (corps jaunes).

TABLEAU 2

Induction artificielle de l'ovulation femelles californiennes allaitantes (I I jours)

(Avril-mai 1971)

\begin{tabular}{|c|c|c|c|c|}
\hline \multirow{3}{*}{ Traitements } & \multirow{2}{*}{$\begin{array}{c}\text { Mâle } \\
\text { vasectomisé }\end{array}$} & \multirow{2}{*}{$\begin{array}{l}\text { Excitation } \\
\text { mécanique }\end{array}$} & \multicolumn{2}{|c|}{ Traitements hormonaux } \\
\hline & & & HCG $25 \mathrm{UI}$ & HCG $50 \mathrm{UI}$ \\
\hline & Lot 1 & Lot 2 & Lot 3 & Lot 4 \\
\hline Nombre de femelles traitées $\ldots \ldots \ldots \ldots \ldots$ & 9 & 16 & 11 & $1 \geq$ \\
\hline Nombre de femelles ayant ovulé .......... & $\overline{\mathbf{s}}$ & 1 & 8 & 11 \\
\hline Nombre de femelles ayant ovulé $(\%) \ldots \ldots$ & 55 & 6 & 72 & 91 \\
\hline Nombre de corps jaunes ............... & 78 & 13 & 108 & 165 \\
\hline Moyenne corps jaune $(m) \ldots \ldots \ldots \ldots$ & 15,60 & & $1: 3,50$ & $\begin{array}{l}15,00 \\
11,44(1)\end{array}$ \\
\hline Variance $\left(\sigma^{2}\right)$ & 1,30 & & $2,(00$ & $\begin{array}{l}76,60 \\
5,27(1)\end{array}$ \\
\hline
\end{tabular}

(1) Valeurs obtenues en éliminant 2 cas de super-ovulation (c.j. $=24$ et 38 ). 


\section{TABLEAU 3}

Induction artificielle de l'ovulation et mortalité embryonnaive à $12 j$ de gestation. Femelles nullipares métisses

(Janvier 1973)

Résultats comparatifs des deux traitements hormonaux

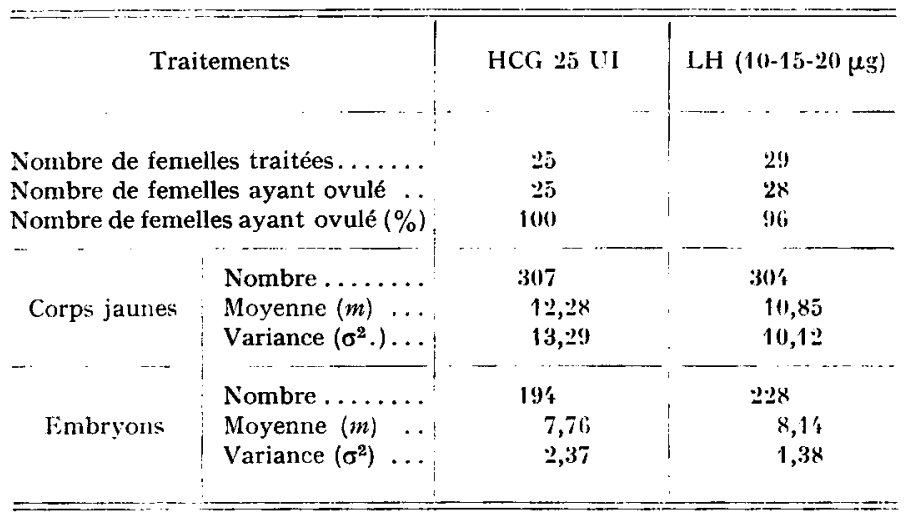

\section{TABLEAU 4}

Mortalité embryonnaire en fonction du nombre de corps jaune. Femelles nullipares métisses

(Janvier r973)

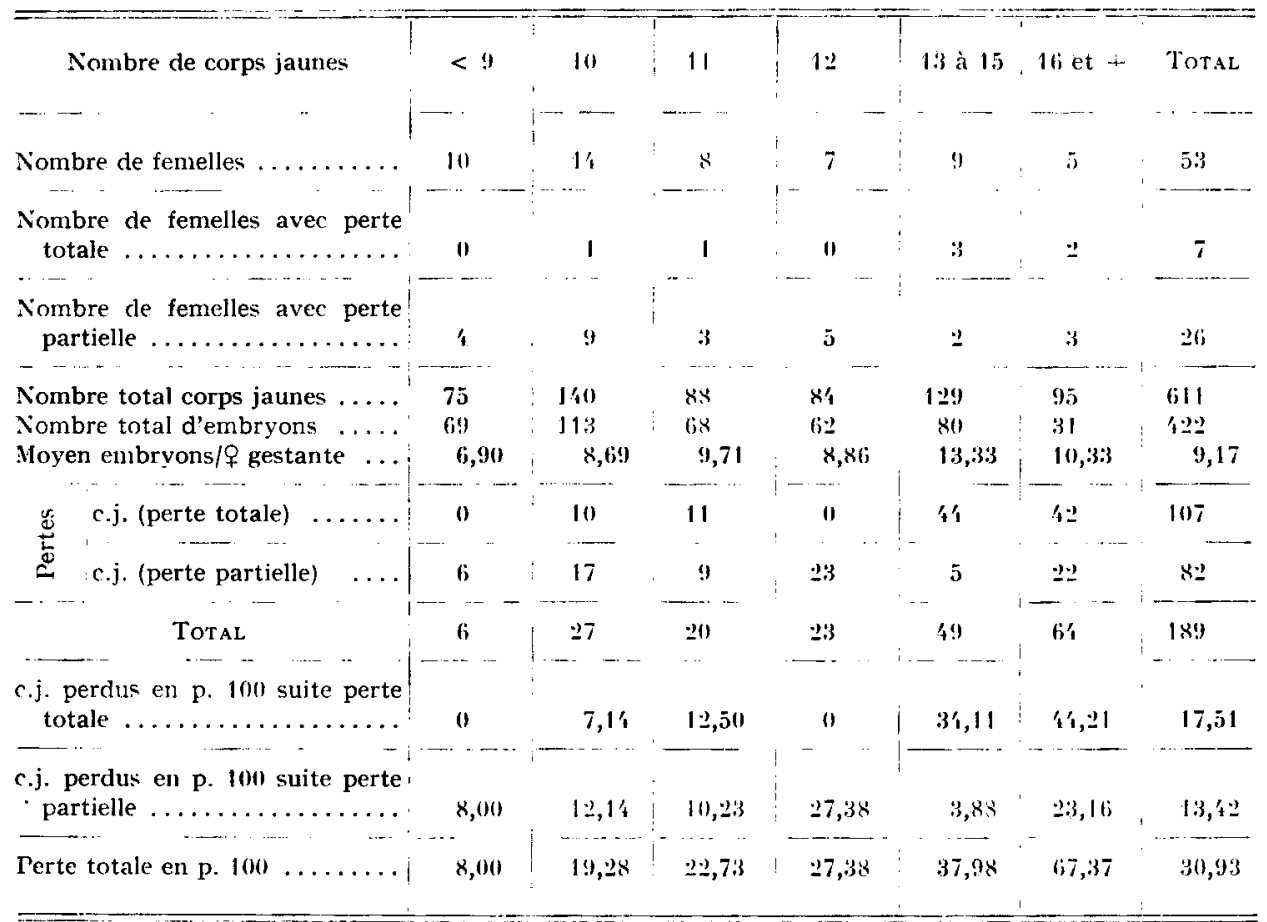


Nombre d'ovulations.

Nous ne considérons ici que les performances des femelles ovulant dans les lots r, 3 et 4 , soit 49 nullipares et 24 allaitantes.

Suite à l'injection de 50 UI d'HCG, nous avons observé parmi les femelles nullipares $(\mathrm{N}=\mathrm{I})$ un cas de superovulation $(37 \mathrm{c.j}$.) et parmi les femelles allaitantes $(\mathrm{N}=\mathrm{II})$ deux cas (24 et $38 \mathrm{c.j}$.). Ces cas ne sont pas considérés dans l'analyse statistique.

Chez les femelles nullipares (tabl. I), le nombre moyen d'ovulations est statistiquement le même pour le lot témoin et les lots traités avec $\mathrm{HCG}(\mathrm{F}=\mathrm{I}, 86$; 0 , Ioo $<p<0,250$ ). Chez les lapines en lactation (tabl. 2) les femelles du lot témoin présentent un nombre d'ovulations supérieur ( $15,6 \mathrm{c.j}$.) à celui des lapines traitées avec $25 \mathrm{UI}$ (I3,5 c.j.) ou 50 UI (II,4 c.j.) de HCG (F=0,29; 0,00I $<p<0,005)$. La différence moyenne de nombres d'ovulations des femelles allaitantes comparées aux femelles nullipares diminue avec l'augmentation de la dose d'hormone : $+4,4 \mathrm{c} . \mathrm{j}$. dans le lot $I,+I, 0$ c.j. dans le lot $3,-0,3$ c.j. dans le lot 4 . La variabilité des résultats par lot ne diffère pas statistiquement pour un état physiologique. L'homogénéité des variances entre lots a été testée par un test $\chi^{2}$ de Bartlett. Elle est réduit'e par rapport aux nullipares, chez les femelles allaitantes pour le lot témoin et le lot traité avec 25 UI d'HCG mais augmente à la suite du traitement à 5o UI d'HCG.

\section{2. - Aptitude des ovocytes à se développer}

Toutes les femelles traitées avec HCG ovulent, une seule présente un défaut d'ovulation avec le traitement $\mathrm{LH}$.

Les valeurs moyennes par traitement (tabl. 3) du nombre d'ovulations et d'enıbryons sont statistiquement égales ( $\mathrm{F}=2,30 ; 0,10<p<0,25 ; \mathrm{F}=\mathrm{I}$, oI $p>0,25$ respectivement), les variances également ( $F=I, 3 I$ et $I, 7 I$ respectivement). Puisque la nature de l'hormone injectée (tout au moins aux doses employées) n'a pas d'effet sur le nombre d'ovulations et le nombre d'embryons, nous étudierons l'aptitude des ovocytes à se développer sur l'ensemble des données. De façon à avoir par classe un effectif suffisant, nous avons regroupé les résultats en fonction du nombre de corps jaunes dénombrés (tabl. 4).

Le nombre d'embryons est inférieur au nombre de corps jaunes. Le déficit d'embryons par rapport à la ponte ovulaire augmente en valeur relative avec cette dernière ; il passe de $8,0 \mathrm{p}$. Ioo chez les femelles présentant moins de Io corps jaunes à $67 \mathrm{p}$. roo chez celles à plus de $\mathrm{I}_{5}$ corps jaunes. Ces pertes résultent d'une part d'un nombre d'embryons inférieur au nombre de corps jaunes chez des femelles gestantes ( 26 cas sur 53 ) d'autre part de l'existence de femelles vides ( 7 cas sur 53 ) bien qu'ayant ovulé. Cinq des sept femelles vides présentaient plus de $\mathrm{I}_{3}$ corps jaunes, la moyenne du nombre de corps jaunes pour ce groupe se situe à I5,29 contre Io,96 chez les femelles gestantes.

\section{DISCUSSION}

L'ovulation peut parfois ne pas avoir lieu à la suite du coit. Nos résultats sur des femelles nullipares et allaitantes le confirment. Ils sont en accord avec les obser- 
vations de différents auteurs qui considèrent que les non-gestations sont le plus souvent liées à des non-ovulations (SAWIN, I954; FoOTE et al., I963), phénomène plus ou moins accentué selon les saisons (HAMmond et MARSHaI.L, I925, FARREI. et al., I968 ; AdAms, I972 ; Selme et Prud'hon, 1973).

I'ovulation peut survenir sans l'intervention du mâle, à la suite d'excitation sexuelle sans pénétration vaginale (HAMMOND, I925; TEMPLETON, I940; BrooK, I937 ; HUloT-RoustaN, non publié) ou après des stimulations mécaniques au niveau des cols utérins (Yamane et Egashiva, I925; Hammond et Asdel., I926 ; Shibata, I93I). Le nombre de femelles ovulant dans ce cas (5 sur 33 traitées) dans notre expérience, est toutefois insuffisant pour que cette technique soit retenue. Ces résultats peuvent être améliorés en traitant auparavant les femelles par des cestrogènes (SAWYER et MARKEE, I959). Ces diverses constatations montrent qu'il est difficile de reproduire artificiellement l'ensemble des stimuli à l'origine du réflexe d'ovulation chez la Lapine et que le taux de réussite est très faible.

Par contre l'injection d'hormones exogènes, chorioniques ou hypophysaires, induit chez des femelles nullipares la ponte ovulaire de façon automatique puisque sur un total de IO2 animaux de toutes races et soumis aux différents traitements, seules 2 femelles n'ont pas ovulé. Les doses les plus faibles utilisées sont dans tous les cas suffisantes. Ce fait est important à souligner car, des quantités élevées de HCG ont parfois été préconisées (MURPHREE et $a l$., I95I : IOo UI).

Sur des femelles primipares allaitantes, nos conclusions seront plus réservées. En effet, le nombre de femelles ovulant après l'injection de HCG est encore élevé (82 p. IOO) mais l'effectif des animaux est rédurt. Nos résultats permettent toutefois d'indiquer que l'induction hormonale artificielle à II jours de lactation est réalisable, fait qui à notre connaissance, n'a jamais été clairement énoncé. Seul un travail d'ADAms (1968) mentionne des ovulations provoquées par HCG chez I7 femelles traitées dans les $24 \mathrm{~h}$ post-partum. Dans notre cas, le pourcentage de lapines qui ovulent n'est pas inférieur à ce qu'il est avec le mâle vasectomisé. Cet acquis est essentiel. Nous considérons que l'étude de l'induction artificielle de l'ovulation est la première étape vers la réalisation de l'insémination artificielle pour obtenir des mises bas groupées sur des lots importants de femelles, celle-ci devant s'effectuer chez des animaux allaitants dans l'optique actuelle d'une accélération des rythmes de reproduction.

En ce qui concerne les femelles ovulant, le nombre moyen de corps jaunes après accouplement ou sous l'influence d'hormones exogènes est comparable chez les femelles nullipares comme l'ont montré les analyses statistiques précédentes. En outre, il est intéressant de noter que ce nombre par femelle est relativement constant, compris pour la plupart entre 9 et I $_{5}$ chez les nullipares. Il existe peu de cas de super-ovulation et la variabilité du nombre de corps jaunes n'est pas accrue. Ira dose de 25 UI HCG semble être suffisante.

Chez les femelles allaitantes, la moyenne du nombre de corps jaunes est plus élevée après saillie $\left(I_{5}, 64\right)$ qu'avec les traitements hormonaux $(13,50$ et $I I, 44)$. La variabilité de ce nombre augmente lorsque la dose d'hormone croît.

L'aptitude des ovocytes à se développer peut être contrôlée par l'importance de la mortalité embryonnaire. Dans notre étude elle sera estimée par excès. In effet nous raisonnons sur une différence entre nombre de corps jaunes, au lieu d'ovules réellement fécondés, et effectif d'embryons. 
L'analyse des résultats conduit à distinguer deux phénomènes : à I 2 jours, I3 p. Ioo des femelles ont des corps jaunes et sont vides. Ces résultats peuvent être comparés à ceux de SELME et PRUD'hon (I973) selon lesquels environ ro p. roo des femelles ayant ovulé après accouplement, ne présentent pas de sites d'implantation. Fox (I968) note des pourcentages de femelles vides à 6 jours relativenent élevés et mentionne des différences entre lots d'animaux accouplés (2I p. IOo) et inséminés artificiellement après injection de HCG (39 p. IOo). Par contre, ADAMs (I960), par accouplement doublé d'une injection de HCG, obtient un pourcentage très bas de femelles vides $(4,3 \mathrm{p}$. IOo). Il note également que le nombre moyen d'ovules chez les femelles vides est anormalement élevé $(I 8,2)$ et qu'une relation existe probablement entre les deux phénomènes. La même remarque peut être faite concernant le résultat de nos expériences où le nombre moyen d'ovules pondus chez les femelles nullipares vides est de 15,25 contre 10,96 chez les femelles gestantes. Cependant le cas des femelles vides ayant un nombre d'ovulations tout à fait normal, reste inexpliqué dans le cadre de cette expérience.

Parmi les femelles gestantes, la moitié des portées est atteinte par des pertes partielles qui représentent $13,5 \mathrm{p}$. Ioo des ovules pondus - en supposant que le nombre d'ovules pondus est égal au nombre de corps jaunes - Ces pertes, selon les animaux, sont très variables et peuvent toucher un seul ovule (40 p. Ioo des femelles) ou la presque totalité de la ponte.

La mortalité embryonnaire a été étudiée chez le Lapin sauvage par ALLEN, BRAMBELI, et MILLs (I947) (saillie naturelle) et chez le Lapin domestique par ADAms (I960) (insémination artificielle doublée de saillie naturelle). Les pertes partielles touchant les femelles gestantes sont importantes : selon ALIEN et al., 43 p. Ioo des ovules pondus seraient perdus au cours de la gestation parmi lesquels Io à I3 p. Ioo disparaîtraient avant l'implantation et environ $20 \mathrm{p}$. Ioo entre IOI 8 jours. Pour ADAms, elles atteignent $30 \mathrm{p}$. Ioo ; un tiers serait perdu avant l'implantation; parmi les deux tiers restants, 7 p. Ioo disparaissent peu après l'implantation, $66 \mathrm{p}$. Ioo entre 8 -I 7 jours et $27 \mathrm{p}$. Ioo entre $\mathrm{I} 7$ et 23 jours.

Le montant des pertes ovulaires partielles (13,5 p. 100) enregistré dans notre expérience semble comparable aux données de ces auteurs, les contrôles ayant été effectués à $\mathrm{I} 2$ jours et couvrant par conséquent le début de la période post- implantatoire.

\section{CONCLUSION}

Chez les lapines nullipares, la ponte ovulaire est induite dans presque tous les cas aussi bien par LH que par HCG. Le taux de gestation suite à une insémination artificielle hétérospermique est inférieur à ce que laisserait espérer le taux de femelles ovulant. Chez I3 p. Ioo des femelles, l'ovulation n'est pas suivie d'une gestation (examen à I2 jours après l'accouplement). En particulier il serait utile de savoir si une femelle n'acceptant pas le mâle mais ayant ovulé sous l'action de la gonadotrophine est apte à être fécondée. Les femelles gestantes paraissent avoir jusqu'à 12 jours une mortalité embryonnaire normale, fait qui impliquerait que les ovules dont la ponte est induite sont dans ce cas susceptibles d'un développement normal. Le fait d'obtenir un pourcentage encore élevé d'ovulation chez les femelles allaitantes 
laisse espérer que cette technique pourrait permettre un rythme de reproduction intensif. Reste posé le problème de la régularité des réponses de femelles subissant périodiquement des injections de gonadotrophines étrangères, pour autant que l'espoir de disposer de $\mathrm{LH}$ de lapin doive être provisoirement écarté.

Reçu pour publication en décembre 1975.

\section{SUMMARY}

ARTIFICIAL INDUCTION OF OVULATION AND FERTILITY

IN THE LACTATING OR NON-I,ACTING RABBIT (ORYCTOLAGUS CUNICULUS)

The number of eggs ovulated following mechanical excitation of the cervix or intravenous $\mathrm{HCG}$ injection ( 25 or $50 \mathrm{IU}$ ) is studied on 72 virgin or 53 lactating California does. Ovaries are examined seven days after treatment. Mechanical stimulation sometimes causes ovulation ( 5 times out of 33). Any experimental amount of hormone injection leads to a high frequency of ovulating animals ( $97 \mathrm{p}$. I 00 of virgin does and $82 \mathrm{p}$. 100 of lactating does). The mean number of eggs ovulated in our sample is quite similar to that observed in virgin does following mating, but lower than for lactating does.

In a second experiment, we study oocyte when ovulation is artificially induced by means of $\mathrm{HCG}$ or ovine $\mathrm{LH}$ injection. Experimental animals are hybrid does of several breeds. The does are slaughtered twelve days after insemination; corpora lutea and embryos are counted. $98 \mathrm{p}$. Ioo of the does ovulate. There is no significant difference in the number of corpora lutea or embryos in the two kinds of induction. The frequency of pregnant does is lower by I3 p. I00 than the frequency of ovulating does. About one-half of the litters show embryonic losses; I 3.5 p. Ioo of eggs ovulated are lost. This figure is similar to that reported in the literature with mating.

\section{RÉFÉRENCES BIBLIOGRAPHIQUES}

Adams C. E., 1960. Studies on prenatal mortality in the rabbit. Oryctolagus cuniculus : the amount and distribution of loss before and after implantation. $J$. Endocr., 19, 325-344.

ADAms C. E., 1968. Ovarian response to human chorionic gonadotrophin and egg transport in the pregnant and post parturient rabbit. J. Endocr., 40, IOI-I05.

Adams C. E., 1972. Induction of ovulation and AI techniques in the rabbit. Vet. rec., 91, $194-197$.

Allen P., Brambell F. W. R., Mills I. H., 1947. Studies on sterility and prenatal mortality in wild rabbits. J.exp. Biol., 23, 312-331.

Brooks C. M., 1937. The role of the cerebral cortex and of various sense organs in the excitation and execution of mating activity in the rabbit. Am. J. Physiol., 120, 544.

Farrel C., Powers D., Otani T., I968. Inhibition of ovulation in the rabbit. Seasonal variation and the effects of indoles. Endocrinology, 83, 599-603.

Foote R. H., Hafs R. E., Staples R. E., Gregolre A. T., Bratton R. W., 1963. Ovulation rates and litter sizes in sexually receptive and non receptive artificially inseminated rabbits given varying dosages of luteinizing hormone. J. Reprod. Fert., $\mathbf{5}, 59-66$.

Fox R. R., 1968. Coitus as an ovulation stimulant in the laboratory rabbit. Proc. Soc. exp. Biol. Med., $128,639 \cdot 642$.

Hammond J., Marshall. F. H. A., I925. Reproduction in the rabbit. 212 p. Oliver and Boyd Edinburgh.

Hammond J., Aspell S. A., 1926. The vitality of the spermatozoa in the male and female reproductive tracts. Brit. J. exp. Biol., 4, I55.

Murphree R. L., Black W. C., Otto G., Casida L. E., r95I. Effects of site of insemination upon the fertility of gonadotrophin treated rabbits of different reproductive stages. Endocrinology, 49, 474-480.

SAwIN P. B., 1954. The influence of age of mother on pattern of reproduction. Ann. N. Y.Acac. Sci., 57, 564-574. 
Sawyer C. N., MarkeE J. E., 1959. Estrogen facilitation of release of pituitary ovulation hormone in the rabbit in response to vaginal stimulation. Endocrinology, 65, 614-621.

Selme M., Prud'hon M., r973. Comparaison, au cours de différentes saisons des taux d'ovulation, d'implantation et de survie embryonnaire chez des lapines allaitantes saillies à l'œestrus post-partum et chez des lapines témoins. Journées de recherches avicoles et cunicoles, I2-1 3-1 4 décembre 1973. 55-58. I.T.A.V.I.

Shibata S., I93I. The oestrus cycle and ovulation in the rabbit. J. Col. Agr., Imper. Iniv. Tokyo, 11, 309-339.

Tempi.eton G.S., 1952. Length of rabbit gestation period. Amer. Rabbit J., 22,93 .

Yamane J., Egashira $T$., 1925. On the relation of copulation to ovulation in the rabbit as shown by means of artificial insemination. J. Jap. Soc. Fet. Sci., 4, I 10. 